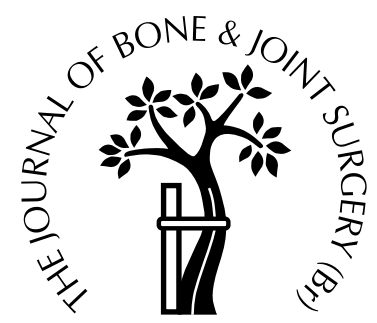

\title{
c-Myc protein in the rabbit growth plate
}

\section{CHANGES IN IMMUNOLOCALISATION WITH AGE AND POSSIBLE ROLES FROM PROLIFERATION TO APOPTOSIS}

\author{
T. Aizawa, S. Kokubun, T. Kawamata, Y. Tanaka, H. I. Roach \\ From Tohoku University School of Medicine, Sendai, Japan and the General Hospital, \\ Southampton, England
}

G

rowth plates taken from five- to 20 -week-old

Japanese white rabbits were immunostained for c-Myc protein. This was localised both in the proliferating zone and upper hypertrophic zone at five weeks, whereas after ten weeks it was found mostly in the lower hypertrophic zone. The proliferating chondrocytes tended to show nuclear staining and the hypertrophic cells cytoplasmic staining, although the terminal hypertrophic chondrocytes sometimes expressed the protein in their nuclei. In the younger rabbits, c-Myc co-localised with proliferating cell nuclear antigen, whereas in the hypertrophic zone of older rabbits, it was present in some chondrocytes the nuclei of which also contained DNA breaks.

Our study suggests that, in the rabbit growth plate, c-Myc is associated with different cellular processes, depending on the age and the developmental stage of the chondrocytes.

J Bone Joint Surg [Br] 1999;81-B:921-5.

Received 16 September 1998; Accepted after revision 29 January 1999

c-Myc protein (c-Myc) is a nuclear associated protein coded for by the proto-oncogene c-myc. ${ }^{1}$ In quiescent cells, there is very little c-Myc. When cells receive mitogenic signals and start to proliferate, c-myc expression is rapidly switched on and c-Myc can be detected in the nuclei. ${ }^{2}$ As cells differentiate, c-myc expression is generally switched off. $^{3}$ Therefore, c-Myc has been thought to be connected with mitogenic stimulation and proliferation. ${ }^{1,3-5}$ In addition, forced constitutive expression of c-Myc prevents terminal differentiation and, ${ }^{4,6}$ in the absence of growth factors, induces apoptosis. ${ }^{7-11}$ This process has been

T. Aizawa, MD, PhD, Lecturer

S. Kokubun, MD, PhD, Professor and Chairman

T. Kawamata, MD, Medical Staff

Y. Tanaka, MD, PhD, University Lecturer

Department of Orthopaedic Surgery, Tohoku University School of Medicine, 1-1 Seiryo-machi, Aoba-ku, Sendai, Japan 980-8574.

H. I. Roach, PhD, University Lecturer

General Hospital, Tremona Road, Southampton SO16 6YD, UK.

Correspondence should be sent to Dr T. Aizawa.

(C)1999 British Editorial Society of Bone and Joint Surgery 0301-620X/99/59492\$2.00

VOL. 81-B, No. 5, SEPTEMBER 1999 studied in fibroblasts, myeloid cells and ovary cells. ${ }^{8-11}$ Thus, c-Myc may be associated with two different cellular processes: cell proliferation and cell death. ${ }^{7,12}$

During endochondral ossification, chondrocytes in the growth plate undergo a complex but well-defined process of differentiation. Cells of the resting zone proliferate initially and then mature to hypertrophic chondrocytes which ultimately undergo apoptosis. ${ }^{13-15}$ In cell cultures of avian embryonic chondrocytes, proliferating cells expressed c-Myc as a transcription factor overexpression of which prevented maturation. ${ }^{4,6}$ This suggested that c-Myc may be involved in maintaining chondrocytes in the proliferating state. In the very active growth plates of threeweek-old chicks and rats c-Myc was localised in the upper hypertrophic zone as well as in the proliferating zone and thus it is also thought to be involved in the differentiation process of chondrocytes. ${ }^{1-5}$ Wang et $\mathrm{al}^{16}$ showed that abundant c-Myc was present in lower hypertrophic chondrocytes of rats around three weeks of age. These studies suggest that the expression of c-Myc may play a more complex role in the growth plate of growing animals than in embryonic chondrocytes, i.e., the expression pattern of cMyc may depend on the developmental stage of chondrocytes in addition to cell proliferation and cell death. ${ }^{16}$ There have been no reports, however, on the changes in localisation of c-Myc with increasing age in vivo. ${ }^{1,4-6}$

Our aim in this study was to investigate the age-related changes in the immunolocalisation of c-Myc in growth plates taken from rabbits of five to 20 weeks of age, the latter being the time near closure of the growth plate. To elucidate the relationship between c-Myc and cell proliferation or apoptosis, we either double-stained for c-Myc and proliferating cell nuclear antigen (PCNA) or c-Myc staining was followed by terminal transferase and deoxyuridine nick-end labelling (TUNEL), ${ }^{17}$ which detects the cells containing DNA strand breaks.

\section{Materials and Methods}

We used 16 Japanese white rabbits. At 5, 10, 15 and 20 weeks of age, four rabbits were killed by intravenous injection of an overdose of pentobarbital sodium. The growth plate was obtained from the middle of the femoral head of each rabbit. 
They were sectioned coronally into blocks $5 \mathrm{~mm}$ thick using a microcutting machine (EXAKT-Apparatebau, Germany) and then fixed in $10 \%$ formalin for three days, decalcified by EDTA at $4{ }^{\circ} \mathrm{C}$ for ten days, embedded in paraffin and cut into slices $2.5 \mu \mathrm{m}$ thick.

Immunohistochemistry. After deparaffinisation and dehydration, the sections from each specimen were either stained with haematoxylin and eosin or immunostained with a monoclonal antibody against human c-Myc (OM11-906A; Genosys, Cambridge, UK). The haematoxylin and eosin sections were used for histological differentiation of the four zones of the growth plate: resting, proliferating and upper and lower hypertrophic. ${ }^{18}$ For immunohistocytochemistry, sections were incubated with the anti-c-Myc antibody at a dilution of $1: 100$ overnight at $4^{\circ} \mathrm{C}$ followed by goat anti-mouse IgG-biotin, extra-avidin peroxidase (Sigma Chemical Company, Poole, UK) and 3-amino 9-ethylcarbazole (AEC) as a substrate. Control sections were incubated without the first antibody but otherwise processed as above. They showed only slight background staining.

Immunolocalisation and quantification of c-Myc expression. c-Myc can be localised in the cytoplasm as well as in the nucleus of chondrocytes. ${ }^{5,16}$ First, we investigated the immunolocalisation of the c-Myc-positive chondrocytes and the intracellular distribution, i.e., whether it was present in the nucleus, cytoplasm or in both. Then, for overall quantification of c-Myc localisation, we scored the chondrocytes with a well-stained cytoplasm or nucleus as positive cells. According to the calculation method of Ueda et $\mathrm{al}^{19}$ for the positive percentage of the Ki-67 index, we counted the positive and negative chondrocytes three times in the five areas containing the largest number of positive cells at a magnification of $\times 200$. The percentage of positive-staining cells was calculated as in our previous study in the whole growth plate and the four individual zones divided due to cell morphology using haematoxylin and eosin staining. ${ }^{20,21}$

Combined stainings. Several sections were double-stained for c-Myc and PCNA. As in our previous study, ${ }^{21}$ the sections were incubated with the antibody to PCNA first, followed by the extra-avidin-biotin method, linked to peroxidase and AEC, which resulted in a brown reaction product. Sections were then incubated with the antibody against c-Myc and visualised using the alkaline phosphatase anti-alkaline phosphatase method (APAAP) with Fast Blue showing a dark blue reaction product. ${ }^{22}$ Control sections were incubated with mouse serum. No positive cells were found in controls.

Another combined staining was performed for the c-Myc and TUNEL method ${ }^{17}$ after sections had been pretreated by microwave irradiation. Twenty slides were irradiated in $200 \mathrm{ml}$ of $0.01 \mathrm{M}$ citrate buffer ( $\mathrm{pH}$ 6.0) for five minutes at a power setting of $50 \%$. This pretreatment enhanced the immunostaining and avoided proteinase $\mathrm{K}$ digestion. Immunostaining of c-Myc was followed by the TUNEL reaction. The incorporated deoxyuridine triphosphate (dUTP)-biotin was detected by binding of avidin-alkaline phosphatase with Fast Blue as a substrate. Negative controls were incubated without c-Myc and terminal transferase. Only background staining was found.

Statistical analysis. The means and standard deviations were calculated for each age group and the four divided zones and statistical evaluation used Fisher's protected least significant difference test. A p value of $<0.05$ was considered significant.

\section{Results}

Chondrocytes staining positively for c-Myc were detected in all four divided zones of all growth plates. The immunolocalisation of c-Myc changed with age. In the resting zone, c-Myc-positive cells were detected in every age group. cMyc showed broad localisation from the resting to hypertrophic zones in the five-week age group with particularly strong staining in the proliferating and upper hypertrophic zones (Fig. 1). Although c-Myc was present both in the nucleus and the cytoplasm, the predominant staining was found within the nucleus in cells of the proliferating zone but also in the cytoplasm of those in the upper hypertrophic zone. At ten weeks of age and later there were few positive chondrocytes in the proliferating zone, whereas many were detected in the hypertrophic zone with c-Myc within the cytoplasm (Fig. 2). Most of the terminal hypertrophic chondrocytes, which were located close to the vascular front, showed positive staining in their nuclei with or without staining in their cytoplasm.

Table I gives the mean numbers of c-Myc-positive chondrocytes in the whole growth plates. The percentage of positive-staining chondrocytes of the five-week age group was significantly higher than that in the other three age groups. The percentages of the individual zones are shown

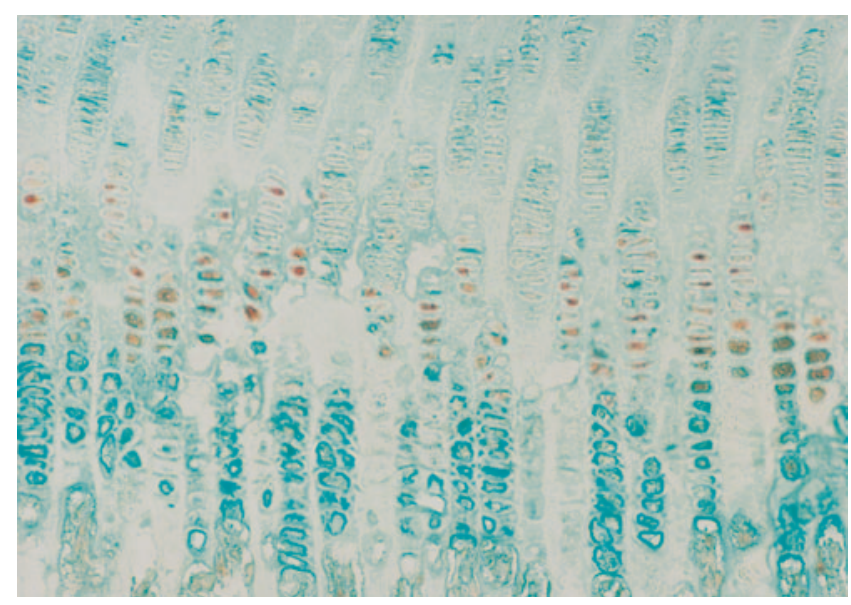

Fig. 1

Immunostaining with c-Myc in a five-week-old rabbit growth plate. Cells with the cytoplasm or nucleus well stained are detected mainly in the proliferating and upper hypertrophic zones $(\times 160)$. 


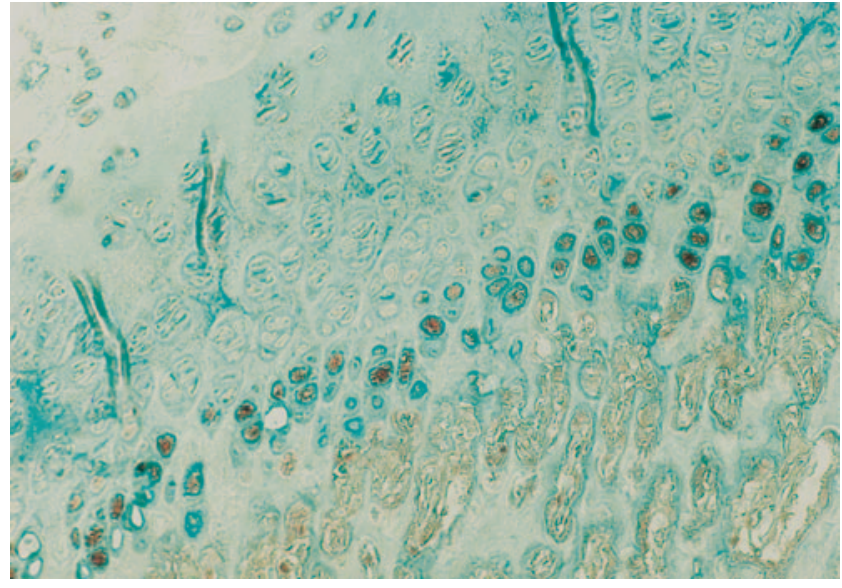

Fig. 2

c-Myc-positive chondrocytes in a 15-week-old growth plate. Many hypertrophic chondrocytes show clear positive staining while chondrocytes in the proliferating zone do not express c-Myc $(\times 160)$.

in Figure 3. In the resting zone, around $10 \%$ of chondrocytes were c-Myc-positive in all age groups. In the proliferating and upper hypertrophic zones, however, a significantly higher percentage of c-Myc-positive chon- drocytes was present at five weeks compared with the older age groups. This contrasts with the findings in the lower hypertrophic zone in which the percentage of c-Myc-positive cells in the five-week age group was significantly lower than in the other three age groups.

In the combined staining for c-Myc and PCNA, c-Myc was present in the nucleus and/or the cytoplasm and PCNA was found in the nucleus. The PCNA-positive cells were detected mainly in the proliferating and upper hypertrophic zones of every age group. In the five-week age group chondrocytes showed positive staining either for c-Myc or PCNA or for both in the proliferating and upper hypertrophic zones (Fig. 4a). In the ten-week-old growth plates, chondrocytes stained with both c-Myc and PCNA were detected as in the five-week age group but the number was reduced. In the 15-week age group, the immunolocalisation of c-Myc and PCNA was clearly separate. The c-Mycpositive cells were detected in the hypertrophic zone whereas the PCNA-positive cells were in the proliferating zone. Few cells were positive for both PCNA and c-Myc (Fig. 4b).

In the combined staining for c-Myc and TUNEL, there were a few cells positive for TUNEL staining in the hyper-

Table I. Mean ( $\pm \mathrm{SD})$ number and percentage of c-Myc-positive chondrocytes at the various stages

\begin{tabular}{lcrrr}
\hline & Weeks & & \\
\cline { 2 - 5 } & $\mathbf{5}$ & \multicolumn{1}{c}{$\mathbf{1 0}$} & \multicolumn{1}{c}{$\mathbf{1 5}$} & \multicolumn{1}{c}{$\mathbf{2 0}$} \\
\hline c-Myc-positive cells & $80.4 \pm 41.7$ & $37.9 \pm 11.6$ & $25.7 \pm 5.9$ & $20.2 \pm 7.4$ \\
Total cells & $512.6 \pm 150.2$ & $439.3 \pm 88.0$ & $310.3 \pm 67.2$ & $231.3 \pm 31.5$ \\
Positive percentage $(\%)$ & $15.2 \pm 4.1^{*}$ & $8.6 \pm 1.7^{*}$ & $8.6 \pm 3.0^{*}$ & $8.7 \pm 2.7^{*}$ \\
\hline$* \mathrm{p}<0.05$ & & &
\end{tabular}

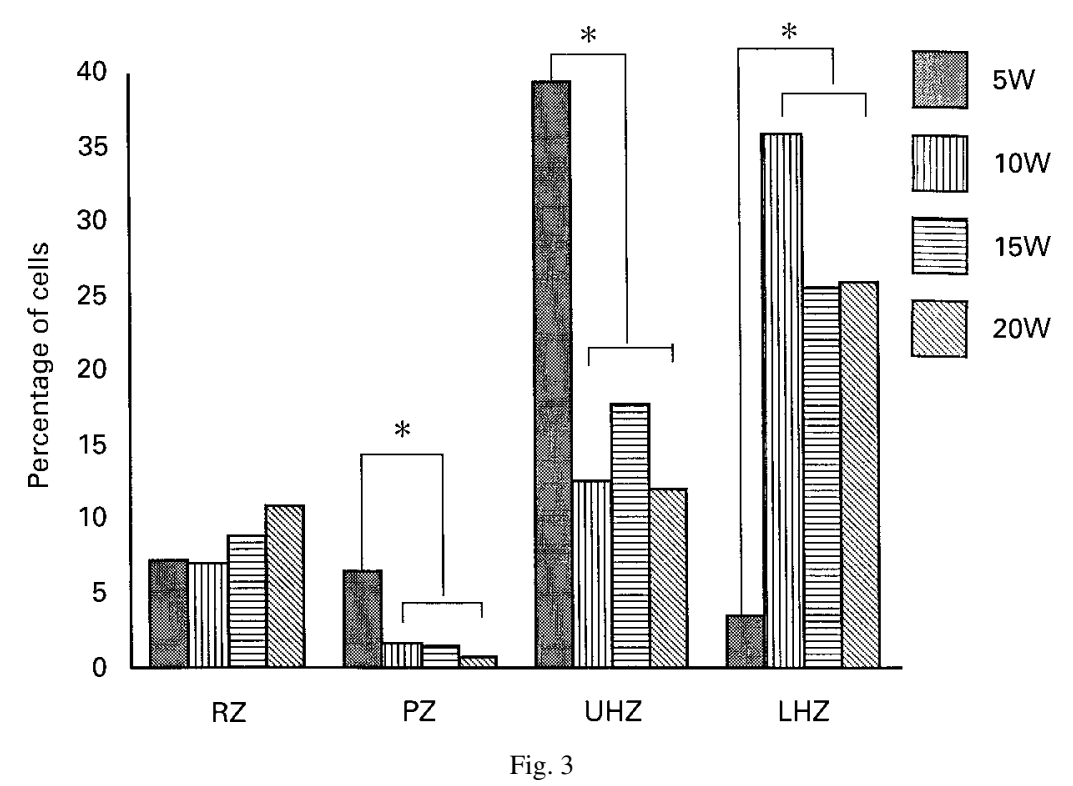

The percentage of positive-staining cells with c-Myc in the four divided zones of the growth plate from five- to 20-week-old rabbits. In the proliferating and upper hypertrophic zones, the percentage of positive cells of the five-week age group is significantly higher than that of the other three age groups. In the lower hypertrophic zone the five-week age group shows a significantly lower incidence than the other age groups (RZ, resting zone; PZ, proliferating zone; UHZ, upper hypertrophic zone; LHZ, lower hypertrophic zone; *, p<0.05). 


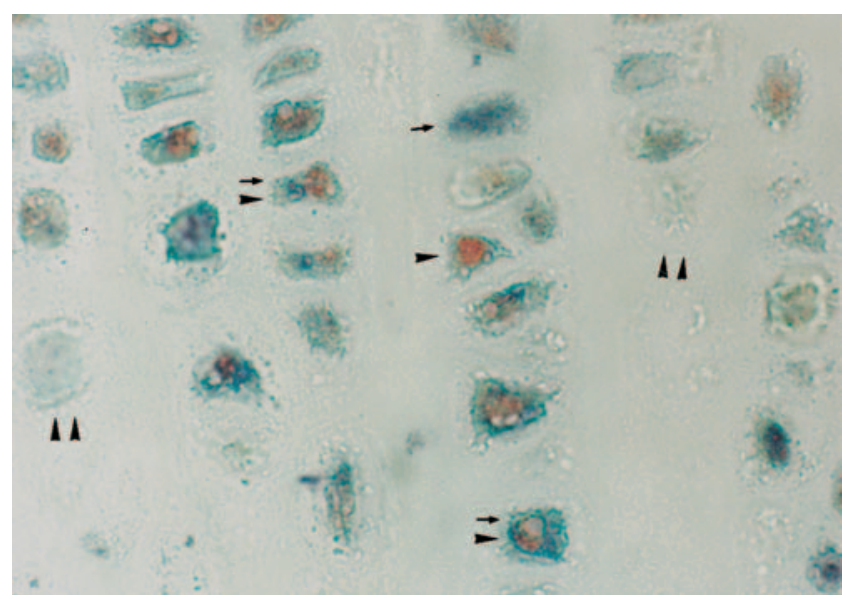

Fig. $4 \mathrm{a}$

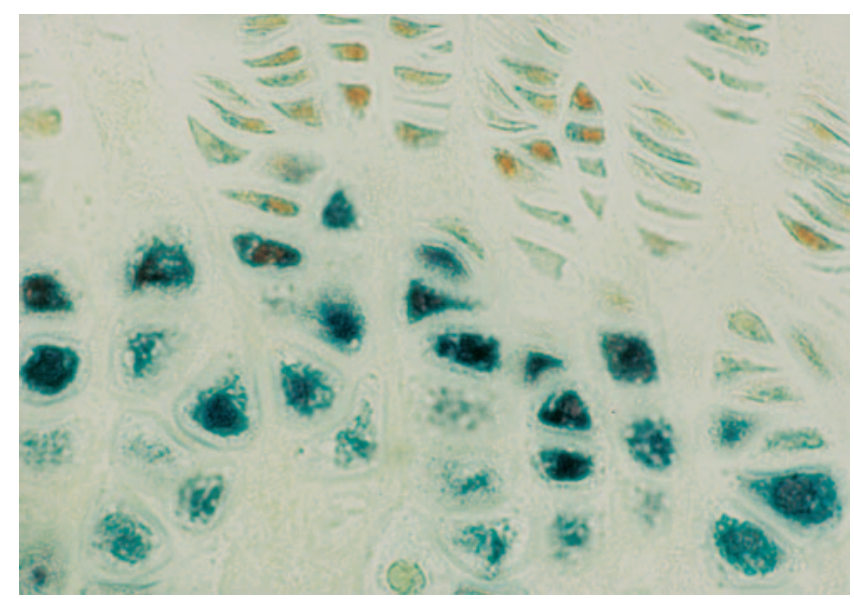

Fig. $4 b$

Combined staining for c-Myc and PCNA. c-Myc-positive cells have a dark blue nucleus and/or cytoplasm and PCNA-positive cells have a brown nucleus. Figure 4a - The proliferating and upper hypertrophic zones at five weeks. Some chondrocytes are positive for c-Myc (arrows), others are positive for PCNA only (arrowheads). There are cells which stain for both (arrows and arrowheads) or have no staining (double arrowheads). Figure 4b - At 15 weeks, c-Myc-positive (dark blue) and PCNA-positive (brown) cells are separately located in the hypertrophic and the proliferating zones, respectively. There are few cells showing positively for both $(\times 390)$.

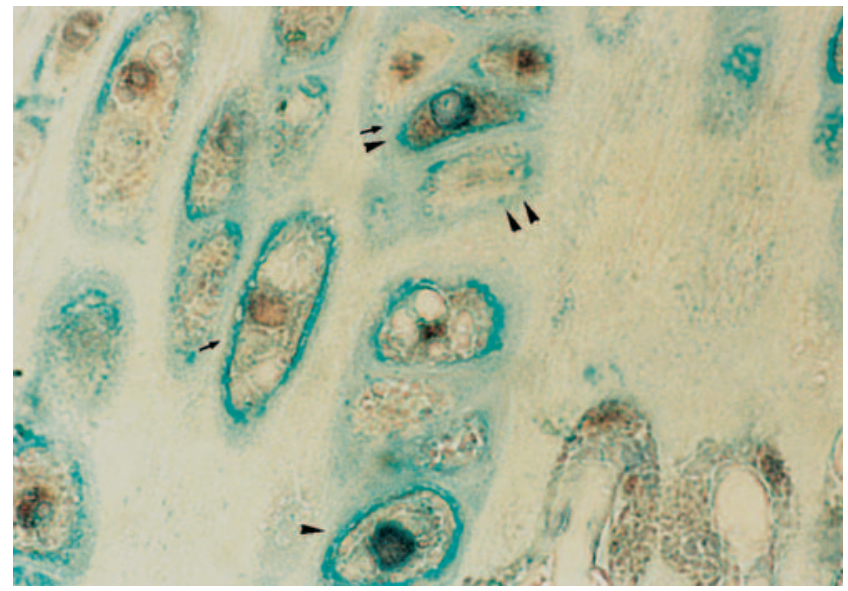

Fig. 5

Combined staining for c-Myc and TUNEL at ten weeks. c-Myc positive cells have a brown nucleus and/or cytoplasm and TUNEL-positive cells have a dark-blue nucleus. Some chondrocytes expressed c-Myc alone in the nucleus and/or cytoplasm (arrows) and others are positive for TUNEL alone (arrowheads). Unstained cells are also present (double arrowheads). There is a chondrocyte with brown cytoplasm showing c-Myc-positive staining and a dark-blue nucleus due to the intensity of the TUNEL reaction (arrows and arrowheads) $(\times 470)$.

trophic zone of the five-week age group. After ten weeks of age hypertrophic chondrocytes were occasionally found in both the upper and lower hypertrophic zones which stained positively, both with c-Myc and TUNEL. Their cytoplasm or nuclei was positive for c-Myc and their nucleus was positive for TUNEL. Chondrocytes labelled with c-Myc alone or TUNEL alone were also present (Fig. 5).

\section{Discussion}

Previous studies have shown that c-Myc was localised in the proliferating and upper hypertrophic zones and therefore it was believed that c-Myc expression in the growth plate of chondrocytes is associated with cell proliferation and differentiation. ${ }^{1,5}$ Additionally, recent reports indicate that $\mathrm{c}-\mathrm{Myc}$ may be an inducer of apoptosis, at least in fibroblasts, myeloid cells and ovary cells. ${ }^{7-11}$ A more recent study suggested that c-Myc expressed in the lower hypertrophic chondrocytes may be associated with apoptosis. ${ }^{16}$ In our study, we have shown changes in the immunolocalisation and intracellular distribution of this protein with age.

In the resting zone, contrary to expectations, c-Myc was detected in some chondrocytes which confirmed the findings of the previous study. Since expression of c-Myc is rapidly increased when quiescent cells are stimulated to proliferate, ${ }^{1}$ its presence in this zone may indicate that these cells have received mitotic stimuli.

In the proliferating zone, many chondrocytes expressed $\mathrm{c}-\mathrm{Myc}$ in the five-week age group, which is in agreement with previous reports. ${ }^{1,5}$ In the combined staining for cMyc and PCNA, there were chondrocytes co-expressing both proteins in the five- and ten-week-old rabbits, confirming a connection between c-Myc and cell proliferation. After ten weeks of age, the percentage of c-Myc-positive cells and the number of chondrocytes co-expressing c-Myc and PCNA was reduced. The proliferating ability of chondrocytes decreases with growth, ${ }^{20}$ which may reflect the decline of c-Myc expression in this zone with increasing age.

In the upper hypertrophic zone, the five-week age group showed a significantly higher positive rate of c-Myc. In the combined staining of c-Myc and PCNA, many chondrocytes stained with both proteins were detected in the fiveweek age group. This suggested that at least some chondrocytes of the upper hypertrophic zone were still proliferating. c-Myc was mainly localised in the cytoplasm, confirming the previous report by Wang et al. ${ }^{16}$ This contrasted with the nuclear localisation in the proliferating zone. While a nuclear localisation was consistent with the 
known role of c-Myc as a transcription factor, the significance of cytoplasmic staining is at present unknown, but may be related to the differentiation of chondrocytes. 5 Very occasionally, chondrocytes in the upper hypertrophic zone were positive for both c-Myc and TUNEL after ten weeks, suggesting that they may undergo apoptosis even in this zone under certain conditions such as mistranscription of DNA. ${ }^{23}$ Hence, in the upper hypertrophic zone, c-Myc may be involved in cell proliferation, differentiation and death.

In the lower hypertrophic zone, the percentage of c-Mycpositive chondrocytes significantly increased after ten weeks. It has already been shown that the rate of apoptosis increases with age in the rabbit growth plate. ${ }^{20}$ After 15 weeks, c-Myc and PCNA no longer co-localised, but were clearly separated with PCNA present in the proliferating zone and c-Myc in the hypertrophic zone, suggesting that the proliferative role of c-Myc decreased with age. By contrast, there was an increased co-localisation of c-Myc with TUNEL-positive chondrocytes, consistent with the known apoptosis-inducing capacity of c-Myc. In most chondrocytes of the lower hypertrophic zone, c-Myc was present in the cytoplasm. In some terminal hypertrophic cells, i.e., those chondrocytes close to the chondro-osseous junction, c-Myc was predominantly present in the nucleus. It is possible that c-Myc may be involved in regulating the transcription of genes associated with apoptosis.

Our study has shown the qualitative and quantitative changes in the expression of the transcription factor c-Myc with increasing age in the rabbit growth plate. It has also suggested that the predominant expression of c-Myc shifted from proliferating to hypertrophic zones which may be connected with the decreased rate of proliferation and increased ratio of apoptosis as the growth plate neared closure.

The authors wish to thank Dr K. Takamatsu and Dr Y. Masumura for help in preparing the material, and also Mr K. Shoji for his dedicated assistance in this study.

No benefits in any form have been received or will be received from a commercial party related directly or indirectly to the subject of this article.

\section{References}

1. Farquharson $\mathbf{C}$, Hesketh $\mathbf{J E}$, Loveridge $\mathbf{N}$. The proto-oncongene cmyc is involved in cell differentiation as well as cell proliferation: studies on growth plate chondrocytes in situ. J Cell Physiol 1992; 152:135-44.

2. Bai MK, Costopoulos JS, Christoforidou BP, Papadimitriou CS. Immunohistochemical detection of the c-myc oncogene product in normal, hyperplastic and carcinomatous endometrium. Oncol 1994;51: 314-9.
3. Meichle A, Philipp A, Eilers M. The functions of Myc proteins. Biochem Biophys Acta 1992;1114:129-46.

4. Iwamoto M, Yagami K, Lu-Valle P, et al. Expression and role of cmyc in chondrocytes undergoing endochondral ossification. $J$ Biol Chem 1993;268:9645-52.

5. Loveridge $\mathbf{N}$, Farquharson $\mathbf{C}$, Hesketh JE, et al. The control of chondrocyte differentiation during endochondral bone growth in vivo: changes in TGF- $\beta$ and the proto-oncogene c-myc. J Cell Sci 1993; 105:949-56.

6. Quarto R, Dozin B, Tacchetti C, et al. Constitutive myc expression impairs hypertrophy and calcification in cartilage. Dev Biol 1992; 149:168-76.

7. Evan GI, Brown L, Whyte M, Harrington E. Apoptosis and the cell cycle. Curr Opin Cell Biol 1995;7:825-34.

8. Harrigton EA, Bennett MR, Fanidi A, Evan GI. c-Myc-induced apoptosis in fibroblasts is inhibited by specific cytokines. EMBO J 1994; 13:3286-95

9. Askew DS, Ashmun RA, Simmons BC, Cleveland JL. Constitutive c-myc expression in an IL-3-dependent myeloid cell line suppresses cell cycle arrest and accelerates apoptosis. Oncogene 1991;6: 1915-22.

10. Evan GI, Wyllie AH, Gilbert CS, et al. Induction of apoptosis in fibroblasts by c-myc protein. Cell 1992;69:119-28.

11. Gibson AW, Cheng T, Johnston RN. Apoptosis induced by c-myc overexpression is dependent on growth conditions. Exp Cell Res 1995; 218:351-8.

12. Farquharson $\mathbf{C}$, Whitehead CC. Differentiation and mineralization in chick chondrocytes maintained in a high cell density culture: a model for endochondral ossification. In Vitro Cell Dev Biol Anim 1995;31:288-94.

13. Farnum CE, Wilsman NJ. Cellular turnover at the chondro-osseous junction of growth plate cartilage: analysis by serial sections at the light microscopical level. J Orthop Res 1989;7:654-66.

14. Lewinson D, Silbermann M. Chondroclasts and endothelial cell collaborate in the process of cartilage resorption. Anat Rec 1992; 233:504-14.

15. Zenmyo M, Komiya S, Kawabata R, et al. Morphological and biochemical evidence for apoptosis in the terminal hypertrophic chondrocytes of the growth plate. J Pathol 1996;180:430-3.

16. Wang Y, Toury R, Hauchecorne M, Balmain N. Expression and subcellular localization of the myc superfamily proteins: c-Myc, Max, Mad1 and Mxi1 in the epiphyseal plate cartilage chondrocytes of growing rats. Cell Mol Biol Noisy Le Grand 1997;43:175-88.

17. Gavrieli Y, Sherman Y, Ben-Sasson SA. Identification of programmed cell death in situ via specific labelling of nuclear DNA fragmentation. J Cell Biol 1992;119:493-501.

18. Robertson WW Jr. Newest knowledge of the growth plate. Clin Orthop 1990;253:270-8.

19. Ueda T, Aozasa K, Tsujimoto M, et al. Prognostic significance of Ki67 reactivity in soft tissue sarcomas. Cancer 1989;63:1607-11.

20. Aizawa T, Kokubun S, Tanaka Y. Apoptosis and proliferation of growth plate chondrocytes in rabbits. J Bone Joint Surg [Br] 1997; 79-B:483-6.

21. Aizawa T, Roach HI, Kokubun S, Tanaka Y. Changes in the expression of Fas, osteonectin and osteocalcin with age in the rabbit growth plate. J Bone Joint Surg [Br] 1998;80-B:880-7.

22. Cordell JL, Falini B, Erber WN, et al. Immunoenzymatic labeling of monoclonal antibodies using immune complexes of alkaline phosphatase and monoclonal anti-alkaline phosphatase (APAAP complexes). $J$ Histochem Cytochem 1984;32:219-29.

23. Wyllie AH, Kerr JF, Currie AR. Cell death: the significance of apoptosis. Int Rev Cytol 1980;68:251-306. 